\title{
Intracavernous de Novo Aneurysms without Prior Manipulation of the Harboring Vessel: Report of Two Cases
}

\author{
Amelie Haase', Stefan Schob², Ulf Quäschling2, Cindy Richter ${ }^{2}$, \\ Jürgen Meixensberger ${ }^{1}$, Ulf Nestler ${ }^{*}$ (i) \\ ${ }^{1}$ Department of Neurosurgery, University Hospital Leipzig, Leipzig, Germany \\ ${ }^{2}$ Department of Neuroradiology, University Hospital Leipzig, Leipzig, Germany \\ Email: *ulf.nestler@medizin.uni-leipzig.de
}

How to cite this paper: Haase, A., Schob, S., Quäschling, U., Richter, C., Meixensberger, J. and Nestler, U. (2020) Intracavernous de Novo Aneurysms without Prior Manipulation of the Harboring Vessel: Report of Two Cases. Open Journal of Modern Neurosurgery, 10, 167-174.

https://doi.org/10.4236/ojmn.2020.101017

Received: December 15, 2019

Accepted: January 5, 2020

Published: January 8, 2020

Copyright $\odot 2020$ by author(s) and Scientific Research Publishing Inc. This work is licensed under the Creative Commons Attribution International License (CC BY 4.0).

http://creativecommons.org/licenses/by/4.0/

(c) (i) Open Access

\begin{abstract}
Intracavernous aneurysms of the cranial base, though less frequent than intradural intracranial aneurysms, can pose considerable therapeutic problems. To date, little is known concerning their spontaneous development and eventual growth kinetics. We report the observation of two de novo aneurysms without prior therapeutic manipulation of the vessels. In our first case, the 33-year-old woman had suffered from an intracerebral hemorrhage at the age of 22. At this time, neurosurgical evacuation was performed, neither angiographically nor histologically was an underlying vascular malformation disclosed. During follow-up observation 11 years later, a cavernous aneurysm of the right internal carotid artery was detected, together with an arteriovenous malformation of the left pericallosal artery next to the former hemorrhage. In the second case, the 49-year-old woman had undergone magnetic resonance imaging (MRI) for headache, revealing two intracavernous aneurysms of the right internal carotid artery. During follow-up, 7 years later, angiography disclosed a de-novo aneurysm of the left internal carotid artery, and an enlargement of the more distal right-sided aneurysm. Most cases of de novo aneurysm formation are described intradurally and after manipulation of the harboring vessel. In the patients presented here, therapeutically induced hemodynamic changes or vessel wall manipulations are not involved, pointing to a cerebrovascular predilection, that-in combination with a set of risk factors-leads to the development of cavernous aneurysms.
\end{abstract}

\section{Keywords}

Aneurysm, Cavernous, De Novo, Incidental, Intracranial 


\section{Introduction}

Incidental intracranial aneurysms are seen in about $3 \%$ of the general population and are increasingly detected due to the vast availability of cranial imaging. Whereas the detection of multiple aneurysms involving several intracranial vessels at first diagnosis is not uncommon, only few patients develop new aneurysms during follow-up of the initial aneurysm. Descriptions of this de novo formation of an aneurysm and introduction of the definition of these lesions as "aneurysms that develop unrelated to the origin of the initial aneurysm" date back to the 1960's [1].

Cavernous aneurysms present a distinct subgroup of intracranial aneurysms, since they predominantly occur extradurally, with a low risk for subarachnoid hemorrhage and rather predispose to carotid-cavernous fistulas or even massive epistaxis [2]. Treatment options comprise occlusion or sacrifice of the parent vessel, but with the advent of flow redirecting devices, vessel wall reconstruction without impeding the cerebral blood supply has become possible [3].

Most observations of de novo aneurysm development report intradural locations beyond the cavernous sinus [4] [5]. In many instances, prior therapeutical manipulation of cerebral vessels had been performed, eventually leading to alterations of cerebrovascular hemodynamics or to disintegration of the endothelial lining, which may trigger aneurysm formation. Identified risk factors comprise female gender, smoking habits, age under 40 years and arterial hypertension. The angiographic detection of multiple intracerebral aneurysms and the location of an aneurysm in the carotid artery both enhance the risk of de novo aneurysm formation [6]. A general predisposition for developing cerebrovascular aneurysms or angiomas during lifetime has been related to diseases such as angiodysplasia or mural degeneration [7] [8].

Though cases without any previous intervention have been described, to the best of our knowledge, no case has been reported with de novo formation of an aneurysm in the cavernous carotid segment. In this article, we describe two cases of cavernous de novo aneurysm formation without prior manipulation of the parent vessel. The first case is related to a cerebral arterio-venous malformation. The second is associated with multiple aneurysms at first diagnosis.

\section{Case Reports}

Case 1

At the age of 22 the female patient had suffered from a frontal pericallosal intracerebral hemorrhage in 2003. Bilateral extraventricular drainages had been placed immediately. After diagnostic angiography had neither disclosed the source of the bleeding nor any vascular malformation, the hematoma had been evacuated neurosurgically by a right frontal craniectomy. Histopathologically, the tissue did not reveal any causes for the bleeding, such as arteriovenous malformation, aneurysm or amyloid angiopathy. The patient recovered completely but did not attend to the scheduled follow-up examinations in our outpatient 
clinic. At 11 years after the bleeding, her general practitioner finally was able to obtain her consent for a cerebral control MRI.

Thus, in 2015 the now 33 year-old woman presented to our outpatient neurovascular unit with routine MRI revealing an arteriovenous malformation (AVM) of the left pericallosal artery, as well as an intracavernous aneurysm of the right carotid artery. This was confirmed by angiography (Figure 1(b)/Figure 1(d)). The aneurysm measured two millimeters in diameter and was of saccular shape. Review of the former angiography confirmed the absence of the aneurysm in 2003 (Figure 1(a)/Figure 1(c)).

Neurosurgical resection of the AVM was advised and for the cavernous aneurysm control MRIs were proposed. During preparation of the surgery, a mild hereditary factor VII deficiency became apparent, with the need for substitution of eptacog alfa and tranexamic acid only before major surgical interventions. With this medications administered, the AVM could be resected uneventfully. Until 2019, follow-up imaging was performed by MRI, according to the patient's preference. The MRIs showed complete resection of the nidus with a small $2 \mathrm{~mm}$ remnant of a pericallosal feeding branch. The size of the right carotid intracavernous aneurysm remained constant over four years. Since the patient still declines angiographic examinations, the next MRI control is planned in February 2020.

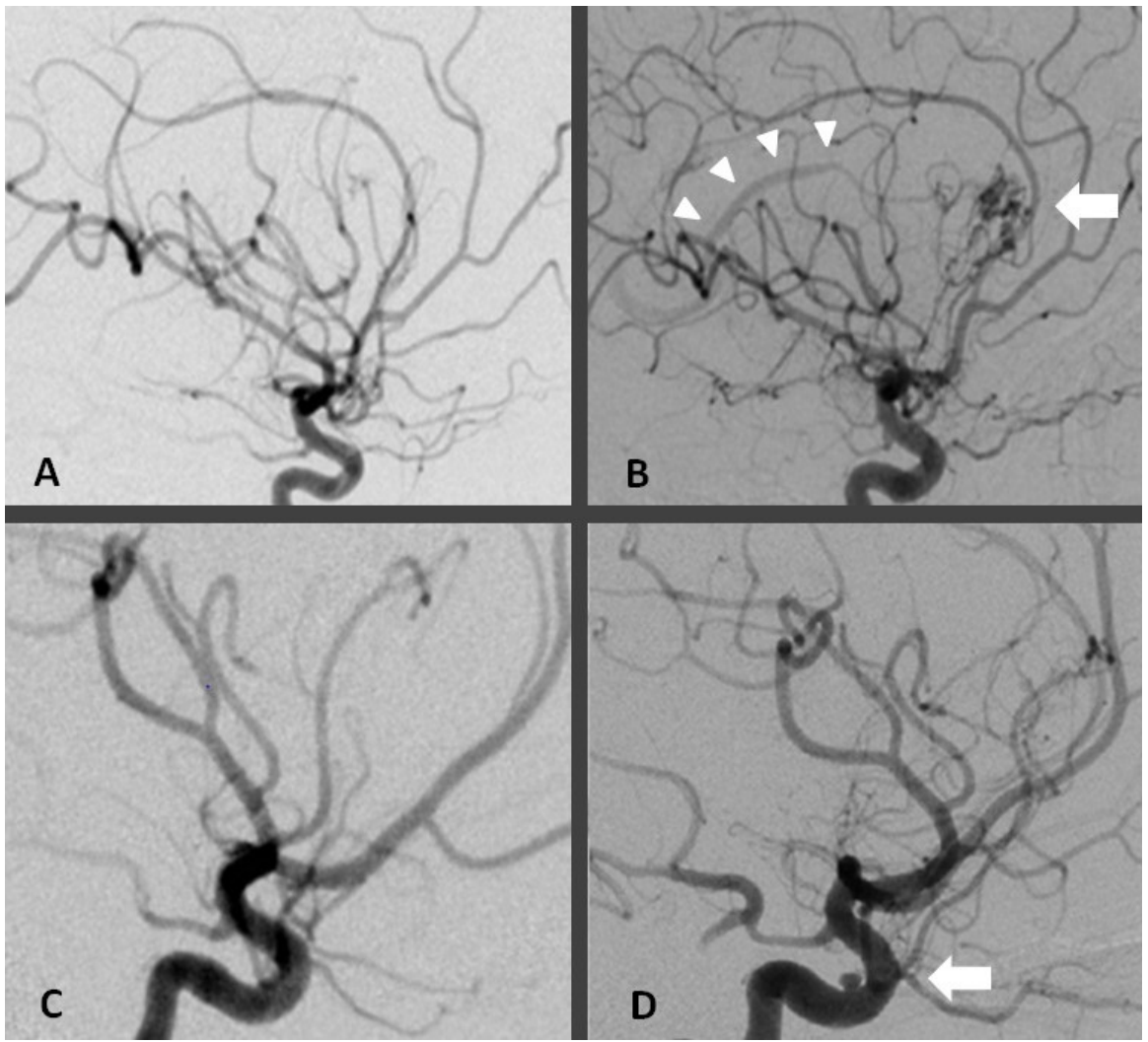

Figure 1. Case 1. (a) and (c) cerebral angiography from 2003, (b) and (d) angiography from 2016. (a) and (b): left pericallosal artery, the arrow points to the angioma nidus, arrowheads depict deep venous drainage. (c) and (d): right internal carotid artery, arrow shows the de novo aneurysm proximal to the ophthalmic artery. 
As anamnestic risk factors for aneurysm development, the patient presented female gender and age under 40, of note, co-occurrence of the arteriovenous malformation was observed.

Case 2

In 2010, at the age of 41, the woman had received a cerebral MRI due to headaches of increasing intensity, resembling to chronic migraine. This MRI had disclosed two intracavernous aneurysms of the right internal carotid artery. Cerebral angiography confirmed a proximal saccular aneurysm with a diameter of four millimeters and a more distal aneurysm with a wide-neck and a diameter of almost two millimeters (Figure 2(c)/Figure 2(d)). The patient had been advised to a conservative management of these aneurysms with regular MR-imaging. The compliance of the patient was very low, she only came back twice to the outpatient clinic, in 2014 without any imaging and in early 2018 with MR-imaging from March 2017. She had not stopped smoking, but at this time point agreed to follow-up angiography.

Eight years after first diagnosis the angiography revealed a mirroring third aneurysm with a diameter of almost $2 \mathrm{~mm}$ in the paraophthalmic segment of the left internal carotid artery (ICA), which had not been present in the previous imaging (Figure 2(a)/Figure 2(b)). In addition, slight enlargement of the

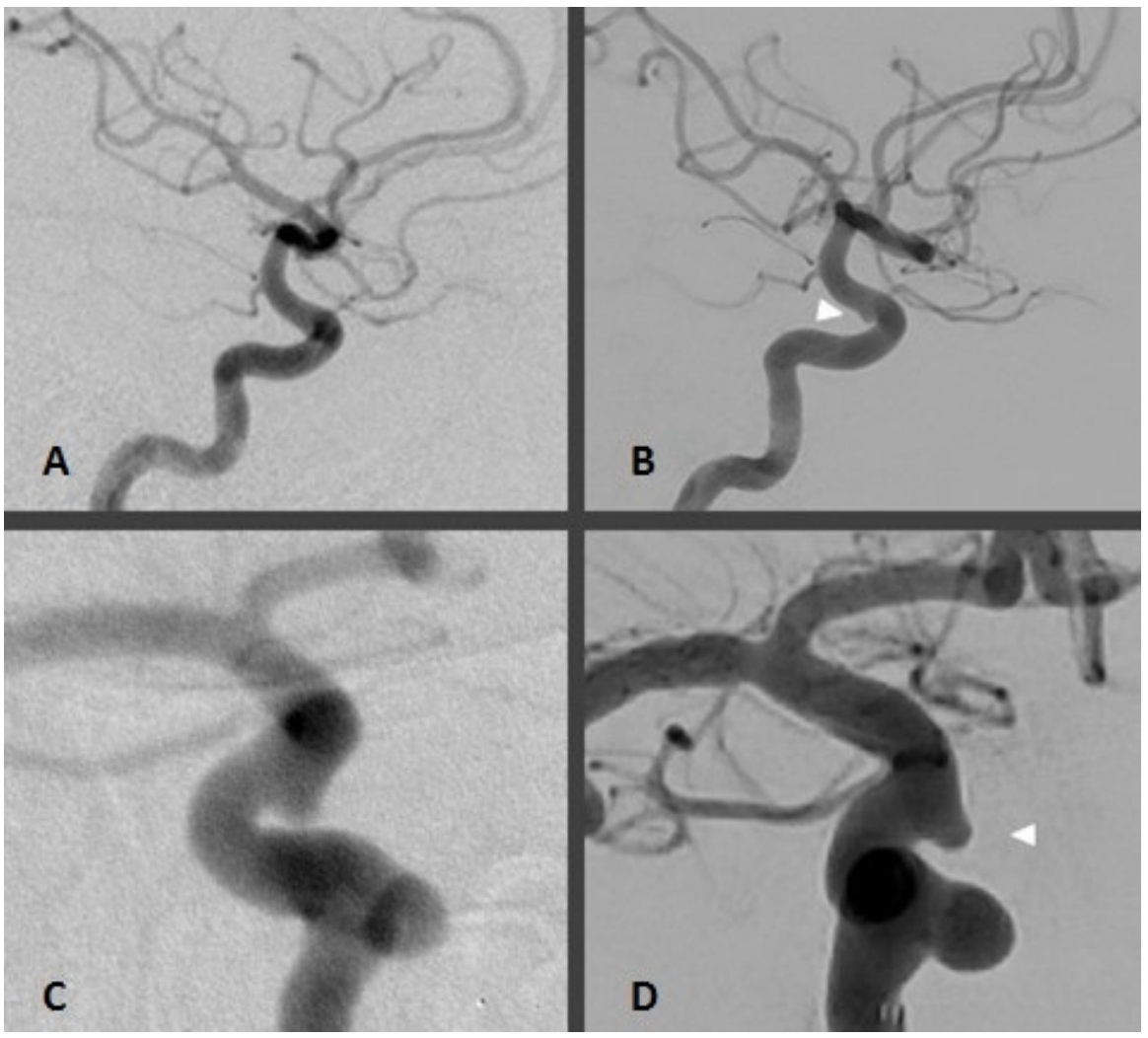

Figure 2. Case 2. (a) and (c) cerebral angiography from 2010, (b) from 2018 and (d) angiography from 2019. (a) and (b): left carotid artery, the arrowhead points to the de novo aneurysm of about $2 \mathrm{~mm}$ size. (c) and (d): right internal carotid artery, the arrowhead shows the distal aneurysm which has slightly increased. 
right-sided distal carotid aneurysm to about 3 millimeters was detected. The proximal aneurysm had remained constant.

Treatment consisted of placing a flow diverter device (p64, Phenox, Bochum, Germany) into the right carotid artery, showing complete occlusion of the distal right ICA-aneurysm in the control angiography one year after the intervention. For the left-sided de novo aneurysm a wait-and-see strategy was chosen. At the last clinical examination in April 2019 the patient did not display any neurologic deficits, she described good improvement of her headaches. The next control angiography is scheduled for March 2020.

The patient's history included female gender, multiplicity of aneurysms and cigarette smoking as vascular risk factors, additional diagnoses were rheumatoid arthritis and Raynaud syndrome.

\section{Discussion}

\section{Patient cases}

Cases of de novo formation of aneurysms without prior manipulation are rare, most reported cases are related to a therapeutic carotid occlusion, potentially altering hemodynamics and biomechanic properties of the arterial wall. In the two cases presented here, de novo aneurysm formation occurred in the cavernous sinus without prior manipulation of the cerebral vascular system. One patient was already harboring intracranial aneurysms; the other patient was simultaneously developing an arteriovenous malformation. Both findings are known to be associated with the formation of de novo aneurysms.

Concerning established risk factors for cerebral aneurysm formation, both patients were female, of young age and the internal carotid artery was involved [6]. Different from patient 1 , patient 2 had multiple aneurysms and a history of smoking, while patient 1 presented with a concurrent AVM.

According to the sequential angiographies, two de novo aneurysm formations and one enlargement of a preexisting incidental aneurysm were observed. Assuming a hypothetical linear kinetic, de novo "aneurysm 1" grew almost 3 millimeters in 11 years ( 0.27 millimeter/year) and de novo "aneurysm 2" reached 2.5 millimeters in 8 years ( 0.31 millimeter/year), allowing for an estimation of a yearly increment rate of 0.29 millimeter/year. The established aneurysm increased about one millimeter in size over a period of 8 years, leading to an increment rate of 0.13 millimeter/year.

\section{AVM and aneurysm}

Arteriovenous malformations (AVM) are known to induce chronic changes in cerebrovascular hemodynamics according to the observation of patients with unruptured intracranial aneurysms and co-existing AVMs [9]. In most cases, these aneurysms develop in the feeders of the AVM. Increased blood flow in the feeding artery is discussed as the main contributor to stress on the vessel wall, which then triggers the formation of aneurysms [10]. Further contributing factors may be lesser resistance in the feeding artery and increased hemodynamic 
turbulences.

Aneurysm location remote from the feeding artery has also been reported. About $43 \%$ of the aneurysms have been described to be without anatomical relation to the AVM feeder and $20 \%$ to be proximal to its origin [11]. None of the aneurysms developed in the cavernous segment of the internal carotid artery.

In our case 1, there is no anatomical proximity between the cavernous aneurysm and the AVM, only a co-occurrence can be noted. Regarding the size of the AVM a flow related induction of the carotid aneurysm seems improbable, whereas a general predisposition of the intracranial vessels to develop wall abnormalities has to be discussed.

There are differing opinions about the ideal treatment strategy for co-existing abnormalities. In most instances, treatment of the aneurysm prior to the treatment of the AVM to prevent enlargement or rupture is suggested. It is assumed that sudden exclusion of the AVM leads to a drastic increase in vascular resistance of the feeding artery, adding a stressor on the aneurysm wall and the nearby upstream arteries. Since these vessels have adapted to the reduced ("stolen") flow, which beforehand drained through the AVM, a breakthrough bleeding can occur [12].

On the other hand, a decrease in size of feeding artery aneurysms after surgically removing the AVM has been observed and the resection of the AVM constitutes an alternative to treat both intracranial lesions [13] [14]. In our case 1, the aneurysm remained constant after resection of the AVM, pointing to local wall abnormalities underlying the development of the aneurysm, rather than to flow related induction.

\section{Multiple intracranial aneurysms}

In a meta-analysis concerning the formation of de novo intracranial aneurysms, multiple intracranial aneurysms at first diagnosis were identified as one of the independent risk factors [6]. This finding had been similarly described in a long-term follow-up study of patients after aneurysm clipping using computed tomography angiography (CTA) [15]. De novo aneurysms can be regarded as a special subset of multiple aneurysms, given the hypothesis of decreased arterial wall shear-stress resistance, affecting parts or even the entire cerebrovascular system [5].

In our case 2, multiple aneurysms had been present before development of the third aneurysm. Two of the three lesions showed an increasing size over 8 years follow-up, consistent with the concept of multifocal shear-stress resistance loss. In addition, continued presence of hemodynamic risk factors such as nicotine consume or arterial hypertension suggest a lifelong process rather than a single event at the origin of the aneurysm formation [16].

\section{Limits of the Study}

Although in both patients the first angiographies did not detect the aneurysms, it remains questionable whether the aneurysm or a small vessel incoherence had 
already been present. Technical factors, individual observer experience or inadequate application of contrast agent can be reasons to miss a small forming aneurysm. Especially the discrimination between overlapping structures can be difficult. Technical advances such as three-dimensional reconstruction of the vessels help to reduce the risk of overlooking small abnormalities. In a follow-up screening after subarachnoid hemorrhage for the frequency of new aneurysms, CT-angiography was performed as imaging strategy. 59 apparently de novo aneurysms were detected and retrospectively; $68 \%$ of these aneurysms had already been detectable on prior CT-angiographies [16].

\section{Conclusion}

Cavernous de novo aneurysm formation without prior manipulation of the harboring vessel is a rarely observed phenomenon, probably associated with a cerebrovascular predisposition for reduced arterial wall resistance. Anatomical factors such as multiplicity of intracranial aneurysms or co-occurrence of aneurysms with cerebral arteriovenous malformations should prompt thorough and long-lasting follow-up using MR-angiography, to enable timely decision-making about safe treatment strategies.

\section{Informed Consent}

Both patients provided written informed consent prior to the therapeutic interventions in 2016 and 2018, respectively, to the use of personal health data for scientific research purposes.

\section{Conflicts of Interest}

The authors declare no conflicts of interest regarding the publication of this paper.

\section{References}

[1] Graf, C.J. and Hamby, W.B. (1964) Report of a Case of Cerebral Aneurysm in an Adult Developing Apparently De Novo. Journal of Neurology, Neurosurgery \& Psychiatry, 27, 153-156. https://doi.org/10.1136/jnnp.27.2.153

[2] Sriamornrattanakul, K. and Akharathammachote, N. (2019) Massive Epistaxis from Non-Traumatic Cavernous Carotid Aneurysm Treated by High-Flow Bypass and Cervical Internal Carotid Artery Ligation: A Case Report and Review of the Literature. World Neurosurgery, 128, 23-28. https://doi.org/10.1016/j.wneu.2019.04.213

[3] Schob, S., Becher, A., Bhogal, P., Richter, C., Hartmann, A., Köhlert, K., Arlt, F., Ziganshyna, S., Hoffmann, K.T., Nestler, U., Meixensberger, J. and Quäschling, U. (2019) Segment Occlusion vs. Reconstruction-A Single Center Experience With Endovascular Strategies for Ruptured Vertebrobasilar Dissecting Aneurysms. Frontiers in Neurology, 10, 207. https://doi.org/10.3389/fneur.2019.00207

[4] Jin, S.C., Choi, C.G. and Kwon, D.H. (2009) Development of “De Novo" Aneurysm after Therapeutic Carotid Occlusion. Journal of Korean Neurosurgical Society, 45, 236-239. https://doi.org/10.3340/jkns.2009.45.4.236

[5] Rinne, J.K. and Hernesniemi, J.A. (1993) De Novo Aneurysms: Special Multiple Intracranial Aneurysms. Neurosurgery, 33, 981-984. 
https://doi.org/10.1227/00006123-199312000-00004

[6] Hu, S., Yu, N., Li, Y., Hao, Z., Liu, Z. and Li, M. (2019) A Meta-Analysis of Risk Factors for the Formation of De Novo Intracranial Aneurysms. Neurosurgery, 85, 454-465. https://doi.org/10.1093/neuros/nyy332

[7] Kagawa, K., Shimizu, H., Matsumoto, Y., Watanabe, M. and Tominaga, T. (2007) Rapid Revascularization after Therapeutic Parent Artery Occlusion for a Large Intracavernous Carotid Artery Aneurysm. Neurologia Medico-Chirurgica (Tokyo), 47, 559-563.

[8] Tonn, J.C., Hoffmann, O., Hofmann, E., Schlake, H.P., Sörensen, N. and Roosen, K. (1999) "De Novo" Formation of Intracranial Aneurysms: Who Is at Risk? Neuroradiology, 41, 674-679. https://doi.org/10.1007/s002340050823

[9] Brown, R.D., Wiebers, D.O. and Forbes, G.S. (2009) Unruptured Intracranial Aneurysms and Arteriovenous Malformations: Frequency of Intracranial Hemorrhage and Relationship of Lesions. Journal of Neurosurgery, 3, 859-863. https://doi.org/10.3171/jns.1990.73.6.0859

[10] Paterson, J.H. and Mckissock, W. (1956) A Clinical Survey of Intracranial Angiomas with Special Reference to Their Mode of Progression and Surgical Treatment: A Report of 110 Cases. Brain, 79, 233-234. https://doi.org/10.1093/brain/79.2.233

[11] Perret, G. and Nishioka, H. (1966) Report on the Cooperative Study of Intracranial Aneurysms and Subarachnoid Hemorrhage-SECTION VI: Arteriovenous Malformations. Journal of Neurosurgery, 25, 467-490. https://doi.org/10.3171/jns.1966.25.4.0467

[12] Batjer, H., Suss, R.A. and Samson, D. (1986) Intracranial Arteriovenous Malformations Associated with Aneurysms. Neurosurgery, 18, 29-35. https://doi.org/10.1227/00006123-198601000-00006

[13] Koulouris, S. and Rizzoli, H.V. (1981) Coexisting Intracranial Aneurysm and Arteriovenous Malformation: Case Report. Neurosurgery, 8, 219-222. https://doi.org/10.1227/00006123-198102000-00012

[14] Hayashi, S., Arimoto, T., Itakura, T., Fujii, T., Nishiguchi, T. and Komai, N. (1981) The Association of Intracranial Aneurysms and Arteriovenous Malformation of the Brain. Case Report. Journal of Neurosurgery, 55, 971-975. https://doi.org/10.3171/jns.1981.55.6.0971

[15] Zali, A., Khoshnood, R.J. and Zarghi, A. (2014) De Novo Aneurysms in Long-Term Follow-up Computed Tomographic Angiography of Patients with Clipped Intracranial Aneurysms. World Neurosurgery, 82, 722-725. https://doi.org/10.1016/j.wneu.2013.06.008

[16] Wermer, M.J.H., Van Der Schaaf, I.C., Velthuis, B.K., Algra, A., Buskens, E. and Rinkel, G.J.E. (2005) Follow-up Screening after Subarachnoid Haemorrhage: Frequency and Determinants of New Aneurysms and Enlargement of Existing Aneurysms. Brain, 128, 2421-2429. https://doi.org/10.1093/brain/awh587

\section{Abbreviations}

AVM-arterio-venous malformation

CT-computed tomography

ICA - internal carotid artery

MRI-magnetic resonance imaging 